\title{
Is hepatotropic contrast enhanced MR a more effective method in differential diagnosis of hemangioma than multi-phase CT and unenhanced MR?
}

Edyta Szurowska ${ }^{1 *}$, Tomasz Nowicki ${ }^{1}$, Ewa Izycka-Swieszewska ${ }^{2}$, Joanna Wypych ${ }^{3}$, Anna Drobinska-Jurowiecka ${ }^{3}$, Karolina Markiet ${ }^{4}$, Arkadiusz Szarmach ${ }^{1}$ and Michal Studniarek ${ }^{1}$

\begin{abstract}
Background: Cavernous hemangiomas are the most frequent neoplasms of the liver and in routine clinical practice they often need to be differentiated from malignant tumors and other benign focal lesions. The purpose of this study is to evaluate whether diagnostic accuracy of magnetic resonance imaging (MRI) of hepatic hemangiomas, showing atypical pattern on US, improves with the use of Gd-BOPTA in comparison with contrastenhanced multi-phase computed tomography (CT).
\end{abstract}

Methods: 178 consecutive patients with ambiguous hepatic masses showing atypical hyperechoic pattern on greyscale US, underwent unenhanced and contrast-enhanced multi-phase multi-detector CT and MR (1.5T) with the use of liver-specific contrast medium gadobenate dimeglumine (Gd-BOPTA). After intravenous contrast administration arterial (HAP), venous-portal (PVP), equilibrium phases (EP) both in CT and MR and additionally hepatobiliary phase (HBP) in MR were obtained. 398 lesions have been detected including 99 hemangiomas and 299 other lesions.

Results: In non-enhanced MDCT examination detection of hemangiomas was characterized by sensitivity of $76 \%$, specificity of $90 \%$, PPV of $71 \%$, NPV of $92 \%$ and accuracy of $86 \%$.

Non-enhanced MR examination showed sensitivity of 98\%, specificity of 99\%, PPV of 99\%, NPV of 99\% and accuracy of $99 \%$.

After intravenous administration of contrast medium in MR the mentioned above parameters did not increase significantly.

Conclusion: Gd-BOPTA-enhanced MR in comparison with unenhanced MRI does not improve diagnostic accuracy in discriminating hemangiomas that show non-specific appearance in ultrasound examination. Unenhanced MR as a method of choice should directly follow US in course of diagnostic algorithm in differentiation of hemangiomas from other liver tumors.

\section{Background}

Cavernous hemangiomas are the most frequent benign neoplasms of the liver, found in autopsy examinations within $0.4 \%$ to $20 \%$ of population $[1,2]$. Such frequent occurrence of hepatic hemangiomas $(\mathrm{HH})$ rises a need for differential diagnosis between those benign lesions

\footnotetext{
* Correspondence: eszurowska@gumed.edu.pl

'Department of Radiology, Medical University of Gdansk, Debinki 7, 80-211 Gdansk, Poland

Full list of author information is available at the end of the article
}

and malignant liver tumors, especially metastases. Many $\mathrm{HH}$ show characteristic image in US examination. However, in some cases hemangiomas show a non-specific appearance in baseline B-mode US [3,4] and contrastenhanced ultrasound (US), computed tomography (CT) or magnetic resonance (MR) examinations are applied for further evaluation of observed focal liver lesions. An atypical hemangioma in US usually shows echogenic border, internal echogenic pattern at least partially hypoechogenic, totally hypoechogenic or heterogenic 
pattern or may contain calcifications or appear as large lesion [5].

Great technological development concerning both multi-detector computer tomography and hepatocytespecific contrast agents in magnetic resonance imaging (MRI) has been observed in recent years.

Computed tomography is a well established procedure of imaging of hepatic hemangiomas. Most hemangiomas show typical enhancement pattern: nodular or globular peripheral enhancement in arterial phase with a progressive fill-in in portal venous and equilibrium phases.

In particular cases the use of magnetic resonance imaging leads to specification of diagnosis based on US and CT findings.

Many studies have been dedicated to differential diagnosis of hemangiomas from malignant tumors [6-8]. Previous studies showed that the use of hepatocyte-specific contrast agents improves the accuracy and confidence of diagnosis of focal liver lesions, however, to our best knowledge none compared gadobenate dimeglumine (Gd-BOPTA) enhanced MRI with unenhanced $\mathrm{MR}$ and CT in differentiation of hemangiomas showing atypical US appearance from other focal liver lesions $[9,10]$. Any imaging technique used for verification of atypical hemangiomas should be validated.

The purpose of this study is to evaluate whether assessment by means of MRI of hepatic hemangiomas, showing atypical appearance on gray-scale US, improves with the use of Gd-BOBTA in comparison with contrast-enhanced multiphase CT.

\section{Methods}

178 consecutive patients with non-specific hepatic foci observed in US were included in this prospective study and spiral CT and MR examinations were performed in those patients within the period not exceeding 3 weeks.

Inclusion criteria were as follows: presence of focal liver lesion that in grey-scale US did not present typical features of a simple cyst (anechoic focus with posterior acoustic enhancement) or a typical hemangioma (hyperechoic oval pattern with homogeneous or slightly inhomogeneous echotexture, well-defined margins and posterior wall shadowing, figure 1a). The atypical hemangiomas included in the study were characterized on US by echogenic border, internal echogenic pattern at least partially hypoechogenic or totally hypoechogenic pattern (figure 1b, c).

Exclusion criteria included any contraindications to CT or MR examination, amongst them contraindications to iodine contrast agents or Gd-BOPTA administration and lack of patient's consent.

The standard of reference was the histopathological examination or clinical and imaging follow-up for the duration of 18 months in case of $\mathrm{HH}$ and $\mathrm{FNH}, 12$ months in case of inflammatory lesions and 3 to 24 months in patients suffering from liver metastases treated with chemotherapy.

MR study was performed with 1.5 T MR system using phased-array flex coil. The protocol included: T1weighted SE sequence (TR/TE ms - 303/12, scan time $17 \mathrm{sec}$ ) in axial and coronal plane with and without contrast enhancement in equilibrium and hepatobiliary phase, T2-weighted Express sequence (18000/92, scan time - 17sec) in axial plane, T2-weighted FSE (6500/ 116.8 , scan time - 20sec) in coronal plane, performed also with fat saturation and T1-weighted out of phase sequence (150/2.24, flip angle of $90^{\circ}$, scan time - 12sec) without and after contrast administration in hepatic arterial dominant phase (HAP), portal venous dominant phase (PVP), equilibrium phase (EP) and hepatobiliary phase (HBP). Slice thickness was $5 \mathrm{~mm}$, intersection gap 0.5 , matrix $256 \times 256$. Dynamic MRI was obtained immediately after a bolus injection of Gd-BOPTA (dosage of $0.1 \mathrm{mmol}$ per kilogram of body weight), followed by saline solution flush of $25 \mathrm{~mL}$ through a $20 \mathrm{G}$ venous catheter positioned in the antecubital vein. Similarly to the multiphase CT study HAP, PVP, and EP were performed after respectively 25, 60 and $180 \mathrm{sec}$. HBP was acquired $60 \mathrm{~min}$ after contrast agent administration. The phaseencoding direction was anterior-posterior for all sequences. All images were acquired during breath-hold.

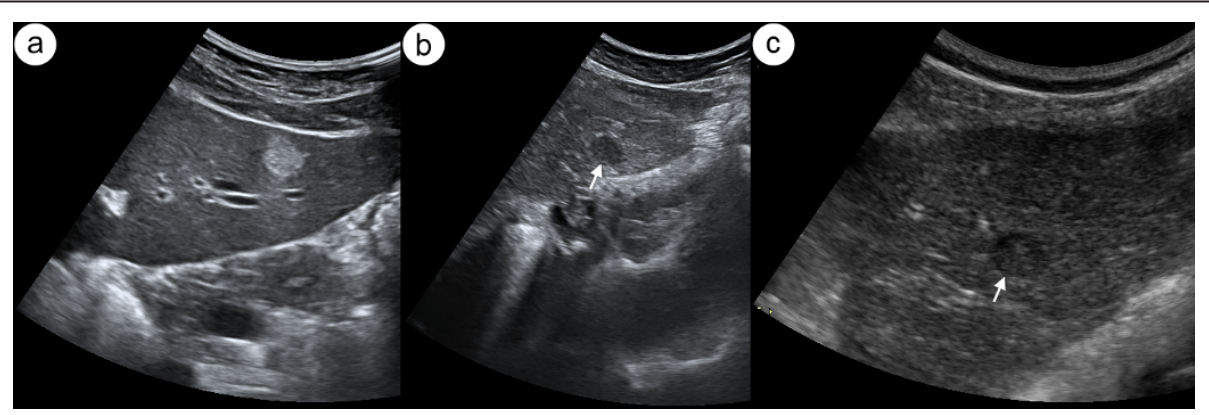

Figure 1 Hepatic hemangioma in US. Transverse sonogram of left lobe of liver presents typical hyperechogenic hemangioma (a). Figure $1 \mathrm{~b}$ and c show two different hypoechogenic liver lesions suspected to be atypical hemangioma, subsequently confirmed in CT. 
Multi-phase multi-detector (6 and 32-row) CT examination of the liver has been conducted first unenhanced, then after application of a contrast agent in HAP, PVP and EP. $100 \mathrm{ml}$ of iodinated contrast agent at a rate of $4 \mathrm{~mL} / \mathrm{sec}$ through an $20 \mathrm{G}$ venous catheter positioned in an antecubital vein using a power injector was administrated. The CT section thickness was $2.5 \mathrm{~mm}$, images interval $-2.5 \mathrm{~mm}$ and pitch - 1.0 .

Spiral CT and MRI were then interpreted by three independent radiologists with at least 10-years (ES, MS) and 5-years (AS) experience in abdominal imaging, who had no previous knowledge of patients' medical history. The interval between CT and MR examinations was from 3 to 5 weeks. Final assessment was based on the observers' consensus in case of disagreement.

Radiological parameters assessed in CT and MR analysis were: size of detectable lesions, their density, signal intensity and type of enhancement.

Signal intensity of each lesion in relation to adjacent liver parenchyma in T2-weighted TSE images was assessed. A 4-point scale has been used: 1-hypointense, 2-isointense, 3-hyperintense and 4-bright lesion (marked hyperintensity, figure 2).

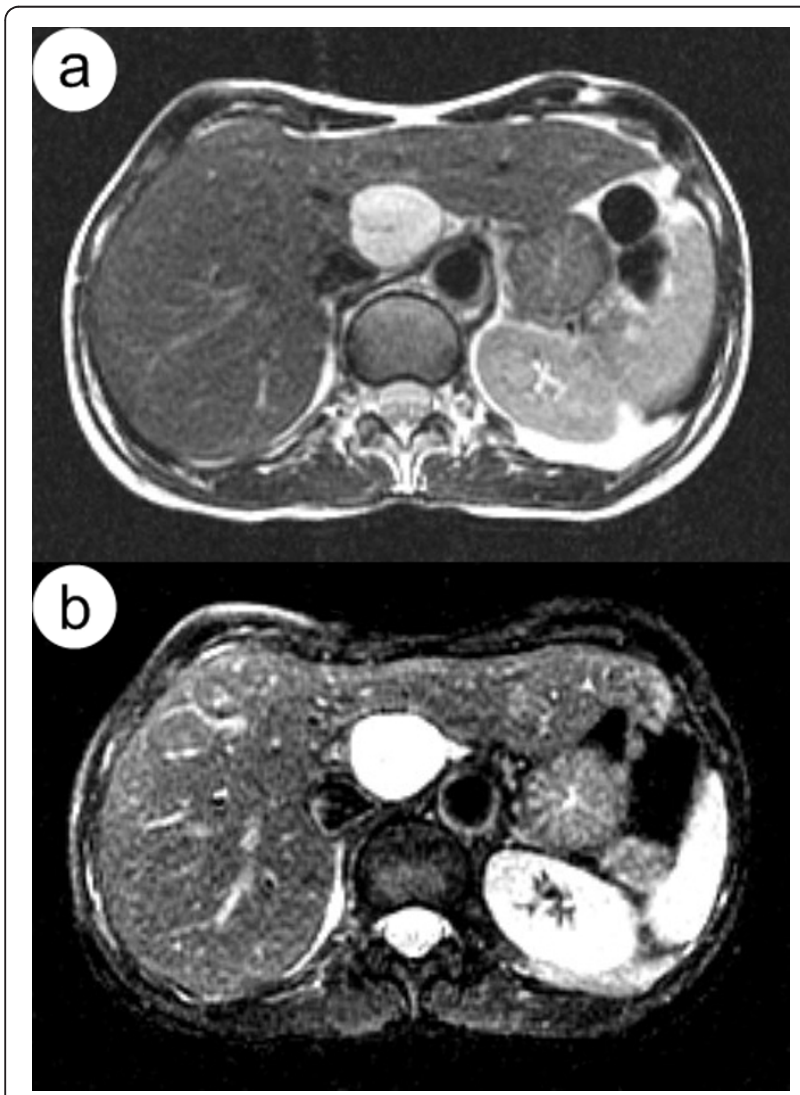

Figure 2 Hepatic hemangioma. Moderately (a) and heavily (b) T2weighted MR images show typical bright lesions.
Type of enhancement in hepatic arterial phase was qualified as one of the following: intensive homogenous, heterogenous, nodular or globular peripheral enhancement, partial or complete ring shape and no enhancement.

In portal venous and equilibrium phase one of the following enhancement type was assigned to each visible lesion: homogenous, heterogenous, progressive fill-in, partial or complete ring shape, no enhancement and enhancement similar to liver.

Each focus was assessed by independent observers in MDCT, unenhanced MRI and Gd-BOPTA-enhanced MRI and classified into one of the groups: hemangioma or non-hemangioma.

In unenhanced MRI hemangioma was defined as a lesion presenting low signal intensity in $\mathrm{T} 1$-weighted images, while in T2- and heavily T2-weighted images remaining highly homogenous, clearly demarcated from the adjacent liver parenchyma, showing very high signal intensity, similar to that of cerebrospinal fluid, due to the long T2 relaxation time of its blood-filled vascular channels. This feature has been described as the bulblight sign.

In dynamic contrast-enhanced CT and MRI hemangiomas were defined when showing peripheral globular enhancement and a centripetal fill-in pattern with the attenuation of enhancing areas corresponding to that of the aorta and blood pool.

Hemangiomas were diagnosed in Gd-BOPTAenhanced MRI when showing typical features of hemangioma in unenhanced-MRI and/or in dynamic contrastenhanced MR examination.

These criteria have been previously applied by many authors [11-14].

Foci were evaluated as of a different type than hemangiomas when not presenting any of the above mentioned features.

All lesions were divided into two groups: the first group ( $\mathrm{HH}$ group) consisted only of liver hemangiomas, the second group (nonHH group) included focal liver lesions other than hemangiomas.

All lesions were also classified as small or large according to their medians by the study coordinator (ES).

The interobserver agreement was measured with Cohen's kappa coefficient. Statistical analysis of size of detectable lesions, their density, signal intensity and type of enhancement of hemangiomas and other lesions in unenhanced, gadolinium-enhanced MR and CT studies has been performed with Statistica 8 software (StatSoft Inc., Tulsa, OK, USA) and McNemar's test with Bonferroni correction for multiply comparison. $P$ values less than 0.05 were considered as statistical significant. 
The research was approved by Independent Bioethic Committee for Scientific Research of Medical University of Gdansk (NKEBN/649/2001-2002). All patients gave their written consent to participate.

The study has been partially financed from the research grant ST-82 given by authorities of the Medical University of Gdansk to MS.

\section{Results}

178 patients were qualified for the study, amongst this group 161 underwent further analysis (95 women and 66 men, age 18-79 years).

In case of 17 patients we failed to establish the final diagnosis - 2 of the patients passed away, 15 did not show for the follow-up.

High interobserver agreement (kappa values of 0.80$0.99)$ confirmed the methodology as reliable and reproducible.

In 100 patients the final diagnosis was based upon histopathological examination results (66 cases by the means of surgery and 34 cases with biopsy) which disclosed: 41 cases of HCC, 21 FNHs, 28 cases of liver metastases, 4 cavernous hemangiomas, 4 solitary adenomas and 2 cases of peripheral cholangiocarcinoma.

In the remaining 61 patients, the final diagnosis was based upon the clinical and diagnostic imaging followup. In this group we observed: 30 hepatic hemangiomas (confirmed in contrast-enhanced US examination and follow-up MR examination), 18 FNHs (confirmed in Gd-EOB-DTPA-enhanced MR), 13 cases of liver metastases (treated with chemotherapy and confirmed in follow-up CT examinations) and 2 inflammatory lesions (follow-up CT examinations showed no lesions).

The HH group consisted of 99 liver hemangiomas, recognized in 34 patients. 21 patients with $\mathrm{HH}$ had isolated liver lesions and multifocal $\mathrm{HHs}$ were recognized in 13 people.

The nonHH group included 127 patients with 299 focal liver lesions other than hemangioma: 144 metastases, 100 cases of hepatocellular carcinoma, 4 adenomas, $44 \mathrm{FNHs}$ and 7 other lesions.

The diameter of foci ranged from $6 \mathrm{~mm}$ to $125 \mathrm{~mm}$ (median $21 \mathrm{~mm}$ ). According to the mean foci diameter, all lesions were divided into two groups: small lesions $(<2 \mathrm{~cm})-199$ foci and large lesions $(\geq 2 \mathrm{~cm})-199$ foci. In the $\mathrm{HH}$-group, 40 foci of cavernous hemangiomas were large lesions and the rest of 59 tumors were small lesions.

Small hemangiomas more frequently (30/59) showed homogenous enhancement pattern in all three phases, while large hemangiomas (25/40) presented nodular or globular peripheral enhancement pattern in HAP and progressive fill-in in PVP and EP in dynamic MR study (table 1). The type of enhancement in consecutive
Table 1 Relation between the enhancement pattern and detectable lesions in HAP and PVP in three groups: small, large and all lesions

\begin{tabular}{|c|c|c|c|c|c|c|c|}
\hline & & \multicolumn{3}{|c|}{$\mathrm{HH}$} & \multicolumn{3}{|c|}{ nonHH } \\
\hline & & small & large & all size & small & large & all size \\
\hline \multirow[t]{5}{*}{ HAP } & homogenous & 30 & 11 & 41 & 46 & 16 & 62 \\
\hline & heterogenous & 1 & 2 & 3 & 1 & 19 & 20 \\
\hline & nodular peripheral & 9 & 25 & 34 & 4 & 7 & 11 \\
\hline & ring shape & 10 & 1 & 11 & 77 & 93 & 170 \\
\hline & no enhancement & 9 & 1 & 10 & 12 & 24 & 36 \\
\hline \multirow[t]{5}{*}{ PVP } & homogenous & 32 & 11 & 43 & 16 & 9 & 25 \\
\hline & heterogenous & 1 & 1 & 2 & 2 & 10 & 12 \\
\hline & progressive fill-in & 9 & 25 & 34 & 2 & 4 & 6 \\
\hline & ring shape & 4 & 1 & 5 & 67 & 81 & 148 \\
\hline & similar to liver & 13 & 2 & 15 & 53 & 55 & 108 \\
\hline
\end{tabular}

phases was completely the same in both $\mathrm{CT}$ and MR studies.

Gd-BOPTA - enhanced MR and multi-phase spiral $\mathrm{CT}$ evaluation of hepatic hemangiomas was based upon typical enhancement pattern in three phases (table 2):

- homogenous in HAP and PVP or EP (figure 3), - nodular peripheral or globular in HAP with progressive fill-in enhancement in PVP and EP (figure 4).

In the hepatobiliary phase of MR examination all hemangiomas showed a lower signal in comparison to the adjacent liver tissue (figure 5). 233 nonHH lesions were hypointense, 63 isointnese and 3 hyperintense.

Spiral CT correctly characterized 75 hemangiomas.

Signal intensity observed in moderately and heavily T2-weighted images in hemangiomas and other liver lesions is presented in table 3.

In unenhanced MR false-negative diagnosis was obtained in one case of hemangioma and one false-positive diagnosis of hemangioma was noted in case of cystic metastasis from ovarian carcinoma.

Gd-BOPTA-enhanced MR study allowed to correctly diagnose cystic metastasis from ovarian carcinoma and therefore revealed 98 hemangiomas. Still one hemangioma was unrecognized (figure 6).

Unenhanced MR and Gd-BOPTA-enhanced MR show higher diagnostic accuracy in differentiation of hemangiomas than CT ( $p<0.0001$ for both modalities), also in case of small $(p<0.0001$ for both modalities) and large lesions assessed separately $(p=0.0055$ for unenhanced MR and $p=0.0009$ for Gd-BOPTA-enhanced MR).

No statistically significant difference in diagnostic efficacy between unenhanced MR and Gd-BOPTAenhanced MR has been showed. 


\begin{tabular}{|c|c|c|}
\hline $\begin{array}{l}\text { Groups and } \\
\text { subgroups }\end{array}$ & $\begin{array}{l}\text { Homogeneous enhancement in HAP, PVP } \\
\text { and EP (number of foci) }\end{array}$ & $\begin{array}{c}\text { Nodular peripheral/globular enhancement in HAP with progressive fill-in } \\
\text { enhancement in PVP and EP (number of foci) }\end{array}$ \\
\hline small HH-group & 30 & 9 \\
\hline $\begin{array}{l}\text { small nonHH- } \\
\text { group }\end{array}$ & 20 & 1 \\
\hline large HH-group & 11 & 25 \\
\hline $\begin{array}{l}\text { large nonHH- } \\
\text { group }\end{array}$ & 5 & 5 \\
\hline $\begin{array}{l}\text { all size } \mathrm{HH}- \\
\text { group }\end{array}$ & 41 & 34 \\
\hline $\begin{array}{l}\text { all size nonHH- } \\
\text { group }\end{array}$ & 25 & 6 \\
\hline
\end{tabular}

The sensitivity, specificity, PPV, NPV and accuracy of unenhanced versus gadolinium-enhanced MR and CT imaging in the characterization of liver hemangiomas are shown in table 4.

\section{Discussion}

Differentiation between hemangiomas and malignant liver lesions is an important clinical problem both in patients with known malignancy as well as in cases of incidentally detected hepatic lesions.

The diagnosis of hemangiomas relies mainly on the following parameters: hyperechogenicity in ultrasound, typical enhancement pattern in CT or MR images (nodular peripheral in HAP with progressive fill-in in PVP and in EP) and high signal intensity in T2-weighted images [11-14].

In dynamic examination the enhancement pattern of hemangiomas does not differ in neither CT nor MR study.

Distinction between metastases and cavernous hemangiomas on the basis of different patterns of enhancement is well described, but to our knowledge, only few reports focus on the type of enhancement, diagnosed with hepatocyte-specific contrast-enhanced MRI [15].

In presented study the assessment of focal lesions in MR was performed with the use of moderately and heavily T2-weighted sequences and dynamic examination after Gd-BOPTA administration.

Ultrasound was the initial examination qualifying patients into this study as it allowed to distinguish a group of patients with ambiguous focal liver lesions, some of suspected malignant nature.

Patients presenting lesions of typical ultrasound appearance of hemangiomas and simple cysts were excluded from further studies as such lesions may be successfully monitored in grey-scale ultrasound examinations.

To assess foci in moderately T2-weighted images qualitative criteria proposed by Fenlon were used [16].
In moderately $\mathrm{T} 2$-weighted images, foci of markedly high signal intensity (so called bright lesions) were classified as hemangiomas (figure 2), assuming that they were homogenous. Lesions with a visible central necrosis were treated as heterogenous and changes in signal characteristics were referred to tissue elements on their periphery. Usefulness of T2-weigthed images for diagnosis of hemangiomas is already well known [7,17-23].

A high diagnostic efficacy of marked hyperintensity in moderately and heavily T2-weighted images as a feature allowing to diagnose hepatic hemangiomas was proven with indexes of diagnostic efficacy reaching 99\% (table 4).

Similar observation was made by Ito et al. [17], who gained $100 \%$ efficacy in discriminating hemangiomas smaller that $3 \mathrm{~cm}$ in diameter and McFarland et al., who reported that dual-echo heavily $\mathrm{T} 2$-weighted sequence $(\mathrm{TE}=80,160 \mathrm{~ms})$ allows to distinguish hemangiomas from malignant neoplasms with $100 \%$ sensitivity and 92\% specificity [18] due to their highly hyperintense signal in T2-weighted images, described as the bulb-light sign [24-26].

Fenlon et al. compared qualitative and quantitative analysis of hepatic tumors using heavily T2-weighted SE technique and they noted that quantitative method with measurement of T2-relaxation times allowed significantly better differentiation between benign and malignant neoplasms with accuracy about $100 \%$ than that of the subjective visual assessment of focal liver lesions [16].

In our series a false-negative diagnosis concerned one case of hemangioma with areas of fibrosis and hyalinization, which mimicked a solid part of malignant necrotic neoplasm. One false-positive diagnosis of hemangioma was reported in case of cystic metastasis from ovarian cancer. We did not observe characteristic strong hyperintensity typical for hemangiomas in other cases of malignant tumors especially in necrotic or hypervascular metastases as noted in literature $[27,28]$. 


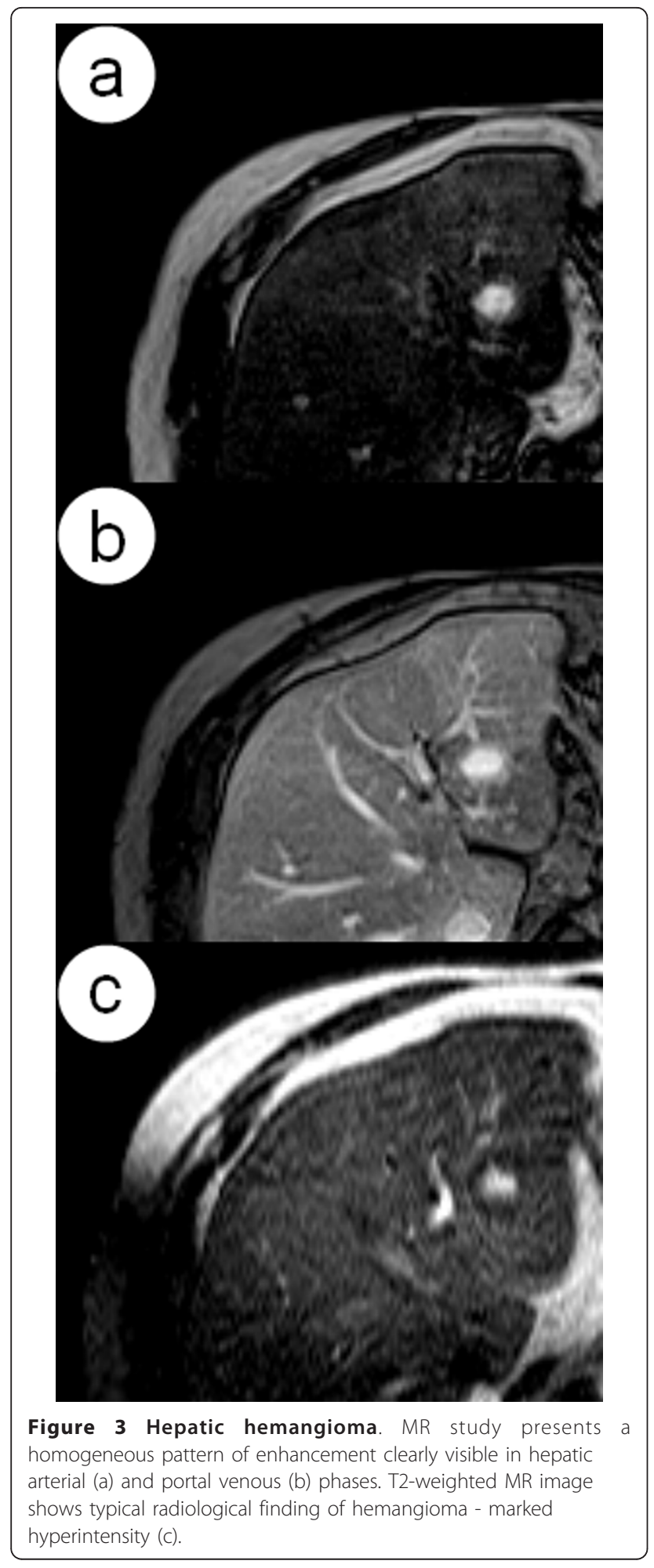

The next radiological feature that allows recognition of $\mathrm{HH}$ is the type of enhancement observed in successive phases of dynamic CT or MR examination after extracellular iodine or gadolinium based contrast medium administration. Ros et al. [29] distinguished three types of enhancement in liver hemangiomas according to their diameter:

- early homogenous enhancement seen in foci of less than $1.5 \mathrm{~cm}$ in diameter,

- nodular peripheral enhancement in HAP with a progressive centripetal fill-in pattern, to homogenous enhancement in the late phase (foci from 1.5 to $5 \mathrm{~cm}$ in diameter),

- nodular peripheral enhancement in HAP with a hypointense center of the lesion in the late phase (foci larger than $5 \mathrm{~cm}$ in diameter).

Essentially in our study two types of enhancement patterns in HAP were stated: nodular peripheral and homogenous (table 1). At the same time correlation between type of enhancement and size of lesion was noted (table 2). In small hemangiomas, homogenous enhancement pattern was observed in HAP, PVP and EP phases in CT and MR studies. This finding was also reported by other authors [30-34] in MR examination and described as flash-filling pattern.

In hemangiomas greater than $2 \mathrm{~cm}$ in diameter a nodular peripheral enhancement pattern in HAP with progressive slow centripetal fill-in during next phases has been observed. This type of enhancement has been described as specific for hemangiomas since 1986 [35-37].

The value of PPV and accuracy and sensitivity in differentiation of hemangiomas from other focal liver lesions based of enhancement pattern presented in multi-phase sCT study are $0.71,0.86$ and 0.76 and noticeably lower than all indexes of diagnostic efficacy obtained by using unenhanced-MRI (PPV-0.99). In the group of lesions smaller than $2 \mathrm{~cm}$, sensitivity of diagnostic methods based on contrast CT enhancement pattern is even lower, at the range of 0.66 .

Presented study in accordance to the other authors $[30,38]$ shows that nodular peripheral enhancement pattern allows discrimination of larger hemangiomas, while it has no application in differentiating small lesions.

In this study all hemangiomas presented weaker enhancement in hepatobiliary phase in comparison to the adjacent liver parenchyma, similarly to metastases and the majority of HCCs. This results from the characteristic of applied hepatocyte-specific contrast media Gd-BOPTA. Gadobenate dimeglumine (Gd-BOPTA) is a gadolinium-based paramagnetic contrast with a vascular-interstitial distribution during first few minutes. The majority of this contrast agent is excreted by the kidneys in the urine, however, about $4 \%$ of the injected dose is taken up by the hepatocytes and eliminated via anionic transporter across the sinusoidal membrane into the bile [39]. 


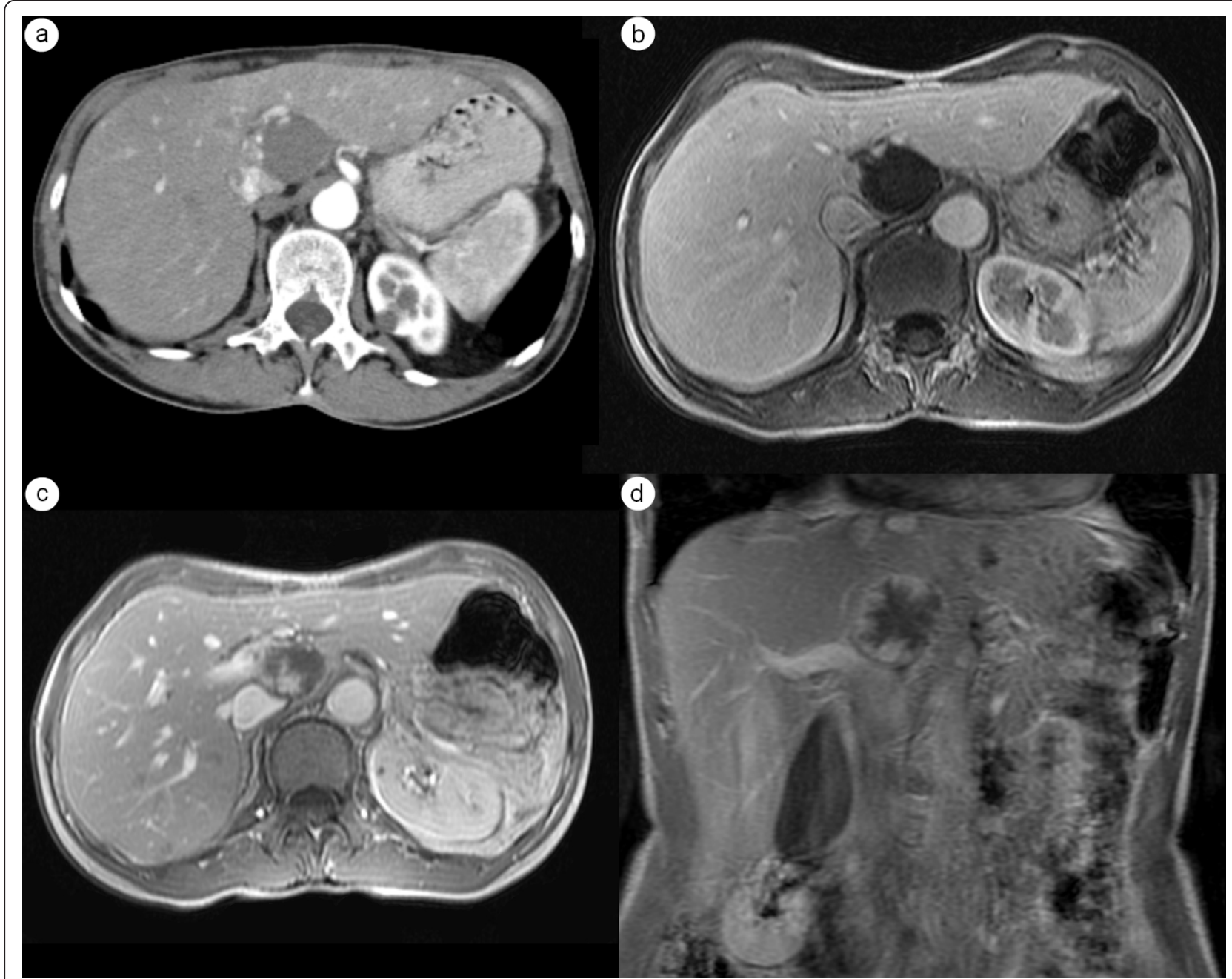

Figure 4 The same case of the hepatic hemangioma as in figure 2. HAP-CT image clearly visualizes globular type of enhancement (a) Globular pattern of enhancement is also visible in HAP - MR image (b). Progressive fill-in enhancement pattern can be observed in PVP (c) and EP (d) phases of MR study.

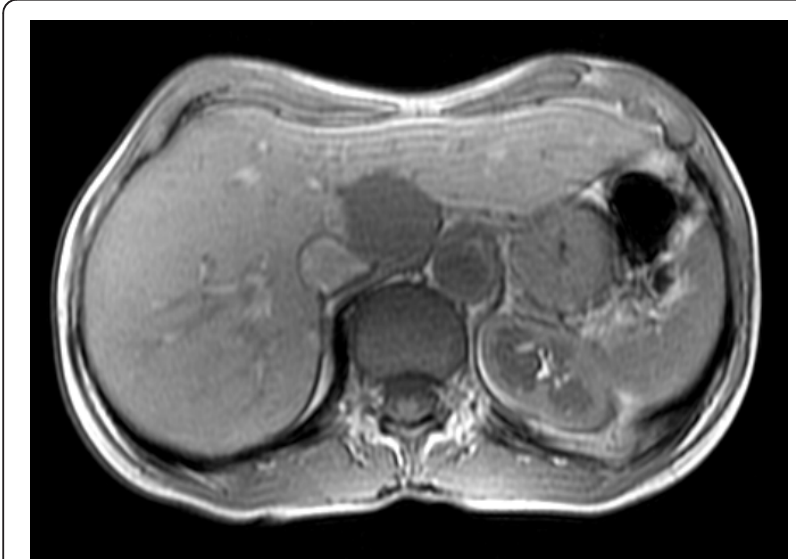

Figure 5 The same case of the hepatic hemangioma as in figures $\mathbf{2}$ and $\mathbf{4}$. Hepatobiliary phase in MR presents weaker enhancement of this lesion in comparison to the adjacent liver parenchyma.
Due to contrast agent presence within hepatic cells, it is possible to observe the enhancement of the liver parenchyma that persists for 1-3 hours after its administration and this period is called hepatobiliary phase.

A tumor that does not contain functioning hepatocytes in which hepatobiliary metabolism is active, is not able to uptake Gd-BOPTA.

Hemangiomas consist of multiple vascular channels limited by single layer of endothelial cells within a thin fibrous stroma and the enhancement in hepatobiliary phase is not observed, what makes hemangiomas impossible to differentiate from other hepatocyte-devoided liver tumors [25,40,41].

Main restrictions of this study are a small number of cases of hemangiomas confirmed in histological examination, lack of quantitative analysis of focal liver lesions signal intensity as described by other authors [42] and 
Table 3 Relation between signal intensity in moderately and heavily T2-weighted images and type of detectable lesions in three groups: small, large and all lesions

\begin{tabular}{lcccc}
\hline & \multicolumn{3}{c}{ signal intensity in moderately and heavily T2-weighted images of all lesions } \\
\cline { 2 - 5 } & hypointense & isointense & hyperintense & markedly hyperintense \\
\hline small HH & 0 & 0 & 0 & 59 \\
\hline small nonHH & 19 & 52 & 1 & 0 \\
\hline large $\mathrm{HH}$ & 0 & 0 & 114 & 39 \\
\hline large $\mathrm{nonHH}$ & 10 & 34 & 1 & 1 \\
\hline all size $\mathrm{HH}$ & 0 & 0 & 183 & 98 \\
\hline all size $\mathrm{nonHH}$ & 29 & 86 & & 1 \\
\hline
\end{tabular}

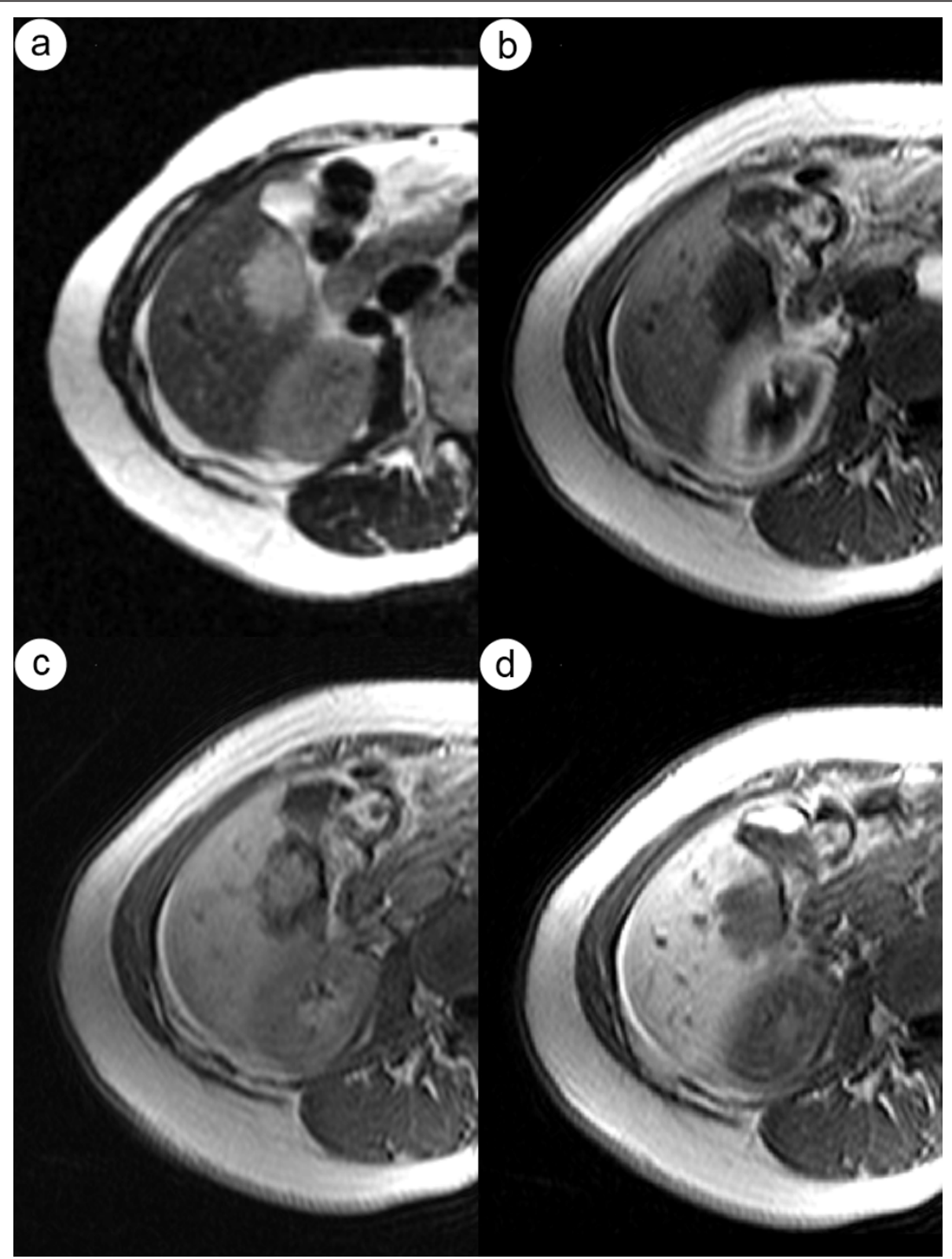

Figure 6 Hepatic hemangioma with atypical enhancement pattern in dynamic MR study. Moderately T2-weighted MR image with untypical weak intensity of the lesion (a). In HAP (b) enhancement of the hemangioma is not visible. Figure $6 \mathrm{c}$ shows central enhancement of the lesion in EP. The hemangioma presents weaker enhancement than the adjacent liver parenchyma in hepatobiliary phase (d). 
Table 4 Reported sensitivity, specificity, PVP, NPV and accuracy of multiphase sCT, Gd-BOPTA-enhanced MR and unenhanced MR studies in the characterization of hemangiomas divided into three groups according to size of diameter: small, large and all lesions

\begin{tabular}{|c|c|c|c|c|c|c|}
\hline & methods & sensitivity & specificity & PPV & NPV & accuracy \\
\hline \multirow[t]{3}{*}{ small lesions } & multiphase sCT & 0.66 & 0.85 & 0.65 & 0.86 & 0.79 \\
\hline & unenhanced MRI & 1 & 1 & 1 & 1 & 1 \\
\hline & unenhanced MRI and Gd-BOPTA-enhanced MRI & 1 & 1 & 1 & 1 & 1 \\
\hline \multirow[t]{3}{*}{ large lesions } & multiphase sCT & 0.9 & 0.94 & 0.78 & 0.97 & 0.93 \\
\hline & unenhanced MRI & 0.97 & 0.99 & 0.94 & 0.99 & 0.98 \\
\hline & unenhanced MRI and Gd-BOPTA-enhanced MRI & 0.98 & 0.99 & 0.95 & 0.99 & 0.99 \\
\hline \multirow[t]{3}{*}{ all size lesions } & multiphase sCT & 0.76 & 0.90 & 0.71 & 0.92 & 0.86 \\
\hline & unenhanced MRI & 0.98 & 0.99 & 0.99 & 0.99 & 0.99 \\
\hline & unenhanced MRI and Gd-BOPTA-enhanced MRI & 0.99 & 1 & 1 & 0.99 & 0.99 \\
\hline
\end{tabular}

no incidence of giant hemangiomas $(>8 \mathrm{~cm})$ in which secondary changes like hemorrhage or hyalinization are more frequent [43].

Our results indicate that qualitative analysis of signal intensity of hepatic lesions in moderately and heavily T2-weighted images is the most sensitive method of differentiation between liver hemangiomas of non-specific appearance in ultrasound from malignant tumors. Assessment of the enhancement pattern provides information about vascularity of the lesion although the type of enhancement pattern itself is not as important in characterization of hemangiomas as the intensity of the lesion in T2-weighted images.

Application of contrast-enhanced MR, especially with administration of liver-specific contrast agents in diagnosing liver hemangiomas is not advisable as it leads to elongation of examination time and unnecessary exposure to potential side effects of intravenous contrast agents.

\section{Conclusion}

Gd-BOPTA-enhanced MR in comparison with unenhanced MRI does not improve diagnostic accuracy in discriminating hemangiomas that show non-specific appearance in ultrasound examination. Unenhanced MR as a method of choice should directly follow ultrasound examination in course of diagnostic algorithm in differentiation of hemangiomas from other liver tumors.

\begin{abstract}
Abbreviations
CT: computer tomography; EP: equilibrium phase; FNH: focal nodular hyperplasia; FSE: fast spin echo; HAP: hepatic arterial dominant phase; HBP: hepatobiliary phase; $\mathrm{HH}$ : hepatic hemangioma; MDCT: multidetector computer tomography; MR: magnetic resonance; MRI: magnetic resonance imaging; nonHH: other lesion than hemangioma; NPV: negative predictive value; PPV: positive predictive value; PVP: portal venous dominant phase; ROI: region of interest; sCT: spiral computed tomography; SE: spin echo; TE: echo time; TR: repetition time; US: ultrasound.
\end{abstract}

\section{Author details}

'Department of Radiology, Medical University of Gdansk, Debinki 7, 80-211 Gdansk, Poland. '2Department of Pathology, Medical University of Gdansk,
Debinki 7, 80-211 Gdansk, Poland. ${ }^{3}$ Department of Gastroenterology and Hepatology, Medical University of Gdansk, Debinki 7, 80-211 Gdansk, Poland. ${ }^{4}$ Department of Neonatal, Gynecological and Urological Radiology, Medical University of Gdansk, Debinki 7, 80-211 Gdansk, Poland

\section{Authors' contributions}

ES has had substantial contribution to study design, data collection and interpretation, statistic analysis and manuscript preparation. TN has contributed to statistic analysis and manuscript preparation. EIS has contributed to data collection and interpretation, as well as manuscript preparation. JW and ADJ has contributed to study design and manuscript preparation. AS has contributed to data collection and interpretation. KM has contributed to manuscript preparation. MS has contributed to study design and has coordinated research team. All authors have read and approved the final manuscript.

\section{Competing interests}

The authors declare that they have no competing interests.

Received: 2 April 2010 Accepted: 19 April 2011 Published: 19 April 2011

\section{References}

1. Goodman ZD, Terracciano LM: Tumours and tumour-like lesions of the liver. In MacSween's pathology of the liver.. 5 edition. Edited by: Burt AD, Portmann BC, Ferrell LD. London: Churchill Livingstone; 2007:795-796.

2. Ishak KG, Rabin L: Benign tumors of the liver. Med Clin North Am 1975, 59:995-1013.

3. Yoon SS, Charny CK, Fong Y, et al: Diagnosis, management, and outcomes of 115 patients with hepatic haemangioma. J Am Coll Surg 2003, 197:392-402.

4. Moody AR, Wilson SR: Atypical hepatic hemangioma: a suggestive sonographic morphology. Radiology 1993, 188:413-417.

5. Vilgrain V, Boulos L, Vullierme MP, Denys A, Terris B, Menu Y: Imaging of atypical hemangiomas of the liver with pathologic correlation. Radiographics 2000, 20(2):379-97.

6. Strobel D, Seitz K, Blank W, Schuler A, Dietrich CF, von Herbay A, FriedrichRust M, Bernatik T: Tumor-specific vascularization pattern of liver metastasis, hepatocellular carcinoma, hemangioma and focal nodular hyperplasia in the differential diagnosis of 1,349 liver lesions in contrastenhanced ultrasound (CEUS). Ultraschall Med 2009, 30(4):376-82.

7. Silva AC, Evans JM, McCullough AE, Jatoi MA, Vargas HE, Hara AK: MR imaging of hypervascular liver masses: a review of current techniques. Radiographics 2009, 29(2):385-402.

8. Heiken JP: Distinguishing benign from malignant liver tumours. Cancer Imaging 2007, 7(Spec No A):S1-14, Review.

9. Petresein J, Spiazzi A, Giovagnoni A, et al: Focal liver lesions: evaluation of the efficacy of gadobenate dimeglumine in MR imaging - a multicenter phase III clinical study. Radiology 2000, 215:727-736.

10. Huppertz A, Balzer T, Blakeborough a, et al: Improved detection of focal liver lesions at Mr imaging: multicenter comparison of gadoxetic acid- 
enhanced Mr images with intraoperative findings. Radiology 2004, 230:266-275.

11. Gibbs JF, Litwin AM, Kahlenberg MS: Contemporary management of benign liver tumors. Surg Clin North Am 2004, 84(2):463-480.

12. Schneider G, Grazioli L, Saini S: Imaging of benign focal liver lesions. In MRI of the liver.. 1 edition. Edited by: Schneider G, Grazioli L, Saini S. Springer-Verlag, Milan, Italy; 2003:105-170.

13. Vilgrain V, Boulos L, Vullierme MP, Denys A, Terris B, Menu Y: Imaging of atypical hemangiomas of the liver with pathologic correlation. Radiographics 2000, 20(2):379-397.

14. Tung GA, Vaccaro JP, Cronan JJ, Rogg JM: Cavernous hemangioma of the liver: pathologic correlation with high-field MR imaging. AJR Am J Roentgenol 1994, 162(2):1113-1117.

15. Kim YK, Lee JM, Kim : Gadobenate dimeglumine-enhanced liver MR imaging: value of dynamic and delayed imaging for the characterization and detection of focal liver lesions. Eur Radiol 2004, 14:5-13.

16. Fenlon $H$, Tello $R$, deCaravalho V, et al: Signal Characteristics of Focal Liver Lesions on Double Echo T2-Weighted Conventional Spin Echo MRI: Observer Performance Versus Quantitative Measurement of T2 Relaxation Times. Journal of Computer Assisted Tomography 2000, 24(2):204-211.

17. Ito K, Mitchell D, Outwater E, et al: Hepatic lesions: discrimination of nonsolid, benign lesions from solid, malignant lesions with heavily T2weighted fast spin echo MR imaging. Radiology 1997, 204:720-37.

18. Lombardo DM, Baker ME, Spritzer CE, Blinder R, Meyers W, Herfkens RJ: Hepatic hemangiomas vs metastases: MR differentiation at 1.5 T. AJR Am J Roentgenol 1990, 155:55-9.

19. Sasaki K, Ito K, Koike S, Fujita T, Okazaki H, Matsunaga N: Differentiation between hepatic cyst and hemangioma: additive value of breath-hold, multisection fluid-attenuated inversion-recovery magnetic resonance imaging using half-Fourier acquisition single-shot turbo-spin-echo sequence. J Magn Reson Imaging 2005, 21(1):29-36.

20. McFarland EG, Mayo-Smith WW, Saini S, Hahn PF, Goldberg MA, Lee MJ: Hepatic hemangiomas and malignant tumors: improved differentiation with heavily T2-weighted conventional spin-echo MR imaging. Radiology 1994, 193:43-7.

21. Outwater EK, Mitchell DG, Vinitski S, Abdominal MR: imaging: evaluation of a fast spin echo sequence. Radiology 1994, 190:425-9.

22. Tang Y, Yamashita Y, Namimoto T: Liver T2-weighted MR Imaging: Comparison of Fast and Conventional Half-Fourier Single-Shot Turbo Spin-Echo, Breath-Hold Turbo Spin-Echo, and respiratory-triggered Turbo Spin-Echo Sequences. Radiology 1997, 203:766-772.

23. Choi BI, Han MC, Kim C-W: Small Hepatocellular Carcinoma versus Small Cavernous Hemangioma: Differentiation with MR Imaging at 2.0 T. Radiology 1990, 176:103-106.

24. Gibbs JF, Litwin AM, Kahlenberg MS: Contemporary management of benign liver tumors. Surg Clin North Am 2004, 84(2):463-480.

25. Schneider G, Grazioli L, Saini S: Imaging of benign focal liver lesions. In MRI of the liver.. 1 edition. Edited by: Schneider G, Grazioli L, Saini S. Springer-Verlag, Milan, Italy; 2003:105-170.

26. Vilgrain V, Boulos L, Vullierme MP, Denys A, Terris B, Menu Y: Imaging of atypical hemangiomas of the liver with pathologic correlation. Radiographics 2000, 20(2):379-397.

27. Namasivayam S, Martin DR, Saini S: Imaging of liver metastases: MRI. Cancer Imaging 2007, 7:2-9.

28. Imam K, Bluemke DA: MR imaging in the evaluation of hepatic metastases. Magn Reson Imaging Clin N Am 2000, 8(4):741-56.

29. Ros PR, Menu Y, Vilgrain V, et al: Liver neoplasms and tumor-like conditons. European Radiology 2001, 11(suppl.2):S145-S165.

30. Kato H, Kanematsu M, Matsuo M, et al: Atypically cavernous hemangiomas: High -resolution gadolinium-enhanced triphasic dynamic gradient-recalled-echo imaging findings. European Radiology 2001, 11:2510-2515.

31. Goshima S, Kanematsu M, Kondo H, Yokoyama Y, Kajita K, Tsuge Y, Shiratori Y, Onozuka M, Moriyama N: Hepatic hemangioma: Correlation of enhancement types with diffusion-weighted MR findings and apparent diffusion coefficients. EJR 2008.

32. Kim KW, Kim TK, Han JK, Kim AY, Lee HJ, Choi BI: Hepatic hemangiomas with arterioportal shunt: findings at two-phase CT. Radiology 2001, 219(3):707-711.
33. Jeong MG, Yu JS, Kim KW: Hepatic cavernous hemangioma: temporal peritumoral enhancement during multiphase dynamic MR imaging. Radiology 2000, 216(3):692-697.

34. Van Hoe L, Bosmans H, Aerts P, et al: Focal Liver lesions: Fast T2-weighted MR imaging with half-Fourier rapid acquisition with relaxation enhancement. Radiology 1996, 2001:8117-823.

35. Freeny PC, Marks WM: Hepatic hemangioma: dynamic bolus CT. AJR 1986, 147:711-719

36. Yamashita Y, Ogata I, Urata J, Takahashi M: Cavernous hemangioma of the liver: pathologic correlation with dynamic CT findings. Radiology 1997, 203:121.

37. Caseiro-Alves F, Bito J, Araujo AE, Belo-Soares P, Rodrigues H, Cipriano A, Sousa D, Mathieu D: Liver haemangioma: common and uncommon findings and how to improve the differential diagnosis. Eur Radiol 2007, 17:1544-1554.

38. Yu JS, Kim MJ, Kim KW, Chang JC, Jo BJ, Kim TH, Lee JT, Yoo HS: Hepatic cavernous hemangioma: sonographic patterns and speed of contrast enhancement on multiphase dynamic MR imaging. AJR Am J Roentgenol 1998, 171(4):1021-5.

39. Pascolo L, Petrovic S, Cupelli F, et al: Abc protein transport of MRI contrast agents in canalicular rat liver plasma vesicle and yeast vacuoles. Biochem Biophys Res Commun 2001, 282:60-66.

40. Huppertz A, Balzer T, Blakeborough a, et al: Improved detection of focal liver lesions at Mr imaging: multicenter comparison of gadoxetic acidenhanced Mr images with intraoperative findings. Radiology 2004, 230:266-275.

41. Petresein J, Spiazzi A, Giovagnoni A, et al: Focal liver lesions: evaluation of the efficacy of gadobenate dimeglumine in MR imaging - a multicenter phase III clinical study. Radiology 2000, 215:727-736.

42. Yamashita Y, Ogata I, Urata J, Takahashi M: Cavernous hemangioma of the liver: pathologic correlation with dynamic CT findings. Radiology 1997, 203:121.

43. Jang H, Kim TK, Lim HK, Park SJ, Sim JS, Kim HY, Lee JH: Hepatic hemangioma: atypical appearances on CT, MR imaging, and sonography. AJR Am J Roentgenol 2003, 180(1):135-141.

\section{Pre-publication history}

The pre-publication history for this paper can be accessed here: http://www.biomedcentral.com/1471-230X/11/43/prepub

doi:10.1186/1471-230X-11-43

Cite this article as: Szurowska et al.: Is hepatotropic contrast enhanced MR a more effective method in differential diagnosis of hemangioma than multi-phase CT and unenhanced MR? BMC Gastroenterology 2011 11:43.

\section{Submit your next manuscript to BioMed Central and take full advantage of:}

- Convenient online submission

- Thorough peer review

- No space constraints or color figure charges

- Immediate publication on acceptance

- Inclusion in PubMed, CAS, Scopus and Google Scholar

- Research which is freely available for redistribution 